\title{
Eletrocardiógrafo digital portátil
}

\author{
Marcelo A. Duarte (UniCarioca) \\ Leonardo O. Ferreira (UniCarioca)
}

\section{Resumo}

Este trabalho teve como objetivo inicial desenvolver uma interface digital, microprocessada, para o projeto "Eletrocardiógrafo de Baixo Custo", desenvolvido por Madureira \& Mendes (2007). O trabalho iniciou-se com o estudo do sistema circulatório, com atenção especial para o coração e seu sistema de condução. Foi realizado um estudo teórico sobre o eletrocardiograma e as principais disfunções cardíacas. Os amplificadores operacionais, principais componentes de todos os estágios analógicos do projeto, e os microcontroladores, cerne do estágio de processamento, também foram estudados e brevemente descritos nesse trabalho. O projeto foi desenvolvido em duas fases distintas: a primeira apresenta algumas melhorias para os circuitos do módulo analógico para detecção, amplificação e condicionamento do sinal elétrico captado diretamente da superfície do corpo humano, em relação ao projeto original de Madureira \& Mendes (2007). Na segunda parte, foi desenvolvido um circuito microprocessado para o tratamento do sinal e para prover uma interface interativa para a visualização do eletrocardiograma. Ao final, os valores teóricos foram sistematicamente comparados com os alcançados pelo protótipo, sendo a viabilidade do projeto discutida, em seguida.

Palavras-chave: Eletrocardiógrafo, Eletrocardiograma, Microcontroladores, Filtros

\begin{abstract}
This work aimed to develop a microprocessed digital interface for the project "Low Cost ECG", developed by Madureira \& Mendes (2007).

The work began by studying the circulatory system, paying special attention to the heart and its conduction system. A theoretical study on the electrocardiogram and the main cardiac dysfunctions was carried out. The operational amplifiers, the main components of all the analog stages of the design, and the microcontrollers, an important part of the processing stage, were also studied and briefly described in this work. The project was developed in two distinct phases: the first presents some improvements to the circuits of the analog module for the detection, amplification and conditioning the electrical signal captured directly from the human body surface, in relation to the Madureira \& Mendes (2007) original project. In the second part, a microprocessed circuit was developed for the signal treatment, and for providing an interactive interface for the electrocardiogram visualization. In the end, the theoretical values were systematically compared with those achieved by the prototype. The feasibility of the project was discussed after this.
\end{abstract}

Keywords: Electrocardiograph, Electrocardiogram, Microcontrollers, Filters

\section{Introdução}

O sistema cardiovascular é composto pelo sangue, pelas artérias, veias, capilares e pelo coração, órgão principal deste sistema. Diversas doenças podem afetar o sistema circulatório, como a fibrilação ventricular e o flutter atrial. A maior parte delas pode ser detectada por um exame simples: o eletrocardiograma (Whittemore, 2009, Parker, 2014). 
O eletrocardiograma é uma importante ferramenta para ajudar no diagnostico de problemas cardiovasculares, pois suas formas de onda contêm informações sobre a natureza das doenças que podem afetar o coração. As formas de onda de um eletrocardiograma são obtidas por intermédio de um equipamento chamado eletrocardiógrafo (Acharya et al., 2007).

Um eletrocardiógrafo, portanto, é um equipamento projetado para coletar a diferença de potenciais corpóreos, em função da atividade cardíaca, apresentando os resultados dessa coleta, geralmente, de forma gráfica (TEB, 2017).

O processo envolvido na captação, amplificação e apresentação gráfica de um sinal cardíaco não é uma tarefa simples. Os potenciais gerados pela atividade cardíaca encontram-se sujeitos ao ruído ambiente e misturados com outros potenciais que emanam da atividade do próprio corpo humano, como por exemplo, o ruído eletromuscular. Além disso, o sinal coletado é de baixa amplitude (da ordem de milivolts) e necessitará de amplificação e filtragem corretas, para que o sinal apresentado ao final do exame esteja de acordo com o que realmente está ocorrendo no coração em análise (TEB, 2017).

Deve-se atentar também para a segurança elétrica atrelada ao uso de um eletrocardiógrafo. Assim, tanto o paciente quanto o médico envolvidos em um exame com esse equipamento devem estar seguros de que nada ocorrerá de diferente, além da coleta dos sinais de interesse. Nesse caso, o equipamento deve garantir, também, que o sinal coletado esteja livre de qualquer ruído e que todo o sinal cardíaco esteja presente no resultado do exame, sem perdas parciais ou totais nas formas de onda necessárias ao estabelecimento de um diagnóstico correto (TEB, 2017).

Com o objetivo de se construir um eletrocardiógrafo preciso e de baixo custo, Madureira \& Mendes (2007) propuseram um modelo analógico, apresentado como trabalho de conclusão de curso na Universidade Gama Filho, em 2007. Tal projeto obteve êxito em diversos aspectos (baixo custo e apresentação de todos os aspectos do sinal cardíaco, por exemplo), ficando aquém do esperado quanto à qualidade do sinal (ainda apresentava ruído na saída) e na apresentação, uma vez que necessitava de equipamento externo para a observação do sinal, não sendo portátil.

O objetivo principal deste novo trabalho foi desenvolver uma interface digital, microprocessada, proposta para o projeto "Eletrocardiógrafo de Baixo Custo", desenvolvido por Madureira \& Mendes (2007), de forma a torná-lo portátil e livre de ruídos. Para isso, o circuito original sofreu alterações, descritas ao longo deste trabalho, para possibilitar um melhor condicionamento do sinal analógico, antes de sua conversão para digital. Além disso, o novo projeto, além de portátil, também garante baixo custo.

Este trabalho, inicialmente, descreve, de maneira sucinta, um estudo sobre o funcionamento e anatomia do sistema circulatório. Logo em seguida, é apresentado um embasamento teórico sobre as principais cardiopatias e distúrbios deste sistema, além do estudo conciso do eletrocardiógrafo. O projeto do eletrocardiógrafo, apresentado mais adiante, foi desenvolvido em duas fases distintas: a primeira apresenta um módulo analógico para detecção, amplificação e condicionamento do sinal elétrico captado diretamente da superfície do corpo humano. Na segunda parte do projeto, foi desenvolvido um circuito microprocessado para o tratamento do sinal, e uma interface interativa para a visualização do eletrocardiograma. Resultados e conclusões são apresentados ao final deste artigo.

\section{Fundamentos teóricos}

\section{Sistema circulatório}

O sistema circulatório é constituído pelo coração, pelo sangue e por um sistema fechado de vasos, que incluem artérias, veias e capilares. Esse sistema carrega oxigênio e nutrientes para todas as células do corpo e capta os resíduos para a degradação e eliminação pelo fígado, pelos rins e pelos pulmões. O bom funcionamento de todos os outros sistemas do corpo humano depende de um sistema circulatório saudável (Whittemore, 2009, Parker, 2014). 
No corpo humano, o sistema circulatório é composto por dois circuitos: o pulmonar e o sistêmico. O pulmonar é suprido pelo lado direito do coração, recebendo o sangue que retorna do corpo, a fim de proceder com a reoxigenação do mesmo. O circuito sistêmico é abastecido pelo lado esquerdo do coração, entregando oxigênio para todas as demais partes do corpo. Em ambos os circuitos, o sangue é pressurizado no coração, trafegando pelos vasos sanguíneos até os capilares, onde realiza as trocas de materiais com as células (Whittemore, 2009, Parker, 2014).

\section{Anatomia do sistema circulatório}

O coração está localizado do lado esquerdo do corpo, na cavidade torácica, entre os pulmões (Figura 1). Como consequência de o coração ocupar um espaço maior do lado esquerdo, o pulmão esquerdo é menor que o direito, possuindo apenas dois lóbulos, enquanto o pulmão direito possui três (Whittemore, 2009, Parker, 2014).

O coração humano possui quatro cavidades. As duas superiores são denominadas átrios e as duas inferiores, denominadas ventrículos. Os lados esquerdo e direito do coração batem como se fossem uma unidade, porém, são separados pelo septo e contêm sangues com características diferentes. Os átrios são separados dos respectivos ventrículos pelas válvulas atrioventriculares (Whittemore, 2009, Parker, 2014).

A função das válvulas é assegurar que o sangue trafegue apenas em uma direção, bloqueando o fluxo reverso. Quando as válvulas falham, seja por estreitamento ou por permitir refluxo, o coração precisa trabalhar mais, o que pode ocasionar um aumento da espessura de sua parede. Essa hipertrofia do coração pode levar ao falecimento do órgão (Acharya et al., 2007).

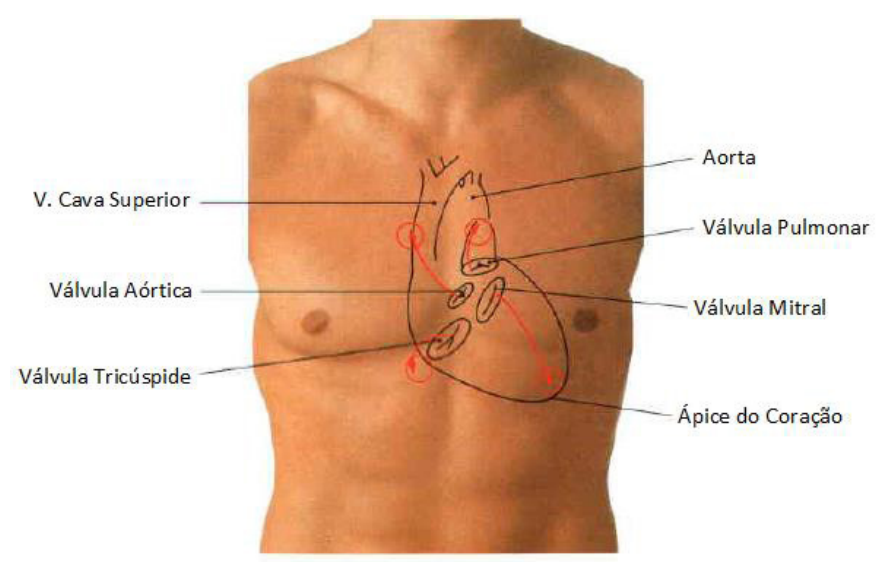

Figura 1 - Localização do Coração no Corpo Humano (SOBOTTA, 2006).

\section{O eletrocardiograma}

O eletrocardiograma é um sinal representativo, que contém informações a respeito da condição do coração. Ele é frequentemente utilizado para avaliar pacientes com problemas cardíacos e é uma importante ferramenta de diagnóstico. A forma e o tamanho das ondas mostradas em um eletrocardiograma (P-QRS-T), vistas na Figura 2, podem conter informações importantes acerca da natureza das doenças que afetam o coração (Acharya et al., 2007).

A presença de anormalidades no sistema circulatório é normalmente refletida no ritmo cardíaco e, portanto, na forma de onda mostrada no eletrocardiógrafo. As características do eletrocardiograma são sempre consideradas para possibilitar um diagnóstico rápido e simples das condições cardíacas (Acharya et al., 2007). 
O eletrocardiograma é produzido a partir de eletrodos conectados em diferentes pontos do corpo. Dependendo da posição destes eletrodos, é encontrado o "ponto de vista” em que o coração é enxergado. Os eletrocardiogramas convencionais apresentam doze "pontos de vista" diferentes, chamados de derivações, obtidos a partir de dez eletrodos. Uma derivação única pode ser produzida a partir de três eletrodos: positivo, negativo e neutro (terra), que fica localizado na perna direita, e que servirá de referência para o equipamento (Acharya et al., 2007).

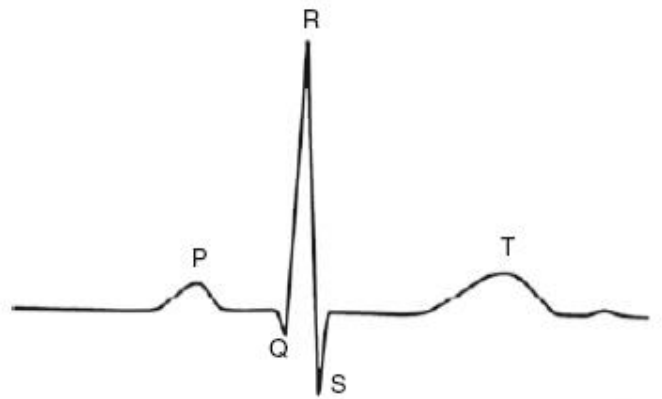

Figura 2 - Ilustração das Ondas P-QRS-T (Macfarlane et al., 2011).

\section{Amplificadores operacionais}

Os amplificadores operacionais estão entre os circuitos mais utilizados na eletrônica. Tais componentes possuem ganho em malha aberta muito alto, normalmente da ordem de 106, impedância de entrada muito alta (alguns $\mathrm{M} \Omega$ ) e impedância de saída muito baixa (poucos $\Omega$ ) (Clayton \& Winder, 2003).

Entre as diversas aplicações executadas pelos amplificadores operacionais, os filtros ativos merecem destaque. Filtros são circuitos eletrônicos capazes de fazer a seleção de frequências, ou seja, eles são capazes de atenuar sinais com frequências indesejáveis e permitir a passagem de sinais com frequências desejadas, com o mínimo de atenuação. Os filtros possuem diversas aplicações no campo da instrumentação, onde a atenuação dos sinais indesejados, como o ruído, é essencial para o funcionamento adequado dos dispositivos (Clayton \& Winder, 2003).

\section{Microcontroladores}

De acordo com Huang (2005), um microcontrolador ( $\mu C)$ é um computador implementado em tecnologia VLSI (Very Large Scale of Integration), que possui, em um único encapsulamento, todos os componentes de um microprocessador, como os registradores, a unidade de controle e a unidade lógica e aritmética (ULA). Além desses, possuem periféricos, como, por exemplo: memórias, timers, conversores Analógico/Digital (A/D) e Digital/Analógico (D/A), comunicação serial assíncrona e síncrona, e comunicação paralela. Apesar de um $\mu \mathrm{C}$ não apresentar uma ULA muito densa, ele possui todos os componentes necessários para um controle de processo, em um único circuito integrado (Souza, 2003).

Os $\mu$ Cs executam as instruções que são gravadas em sua memória. Essas instruções são, na verdade, números binários chamados de código de máquina. Escrever um programa em código de máquina é uma tarefa pouco eficiente, uma vez que o programador precisa consultar tabelas constantemente. $\mathrm{O}$ assembly foi criado para simplificar o trabalho do programador. As instruções em assembly são representações do código de máquina. Ele revolucionou a tarefa do programador, uma vez que este não mais precisava se basear em 
códigos binários, mas em uma linguagem um pouco mais próxima da sua realidade (Huang, 2005).

O uso do assembly apresenta algumas desvantagens: o programador necessita de um amplo conhecimento do hardware do $\mu \mathrm{C}$, e a produtividade é baixa, sobretudo para programas extensos, já que o programador necessita trabalhar com lógica de baixo nível. Com o intuito de facilitar o programador, foram desenvolvidas linguagens que trabalham com lógica de alto nível, como por exemplo, as linguagens $\mathrm{C}$ e o Basic. As linguagens de alto nível são bem próximas da língua inglesa e, portanto, muito mais fáceis de serem compreendidas. Uma instrução em linguagem de alto nível pode conter até centenas de instruções de baixo nível. O uso de compiladores específicos permitirá a tradução de um programa projetado em linguagem de alto nível, para uma linguagem de baixo nível e/ou para o código de máquina correspondente.

\section{Materiais e Métodos}

O objetivo inicial deste trabalho era apenas desenvolver uma interface digital, microprocessada, para o projeto "Eletrocardiógrafo de Baixo Custo", desenvolvido por Madureira \& Mendes (2007). Porém, foram realizadas, no circuito original, algumas alterações para possibilitar um melhor condicionamento do sinal analógico, antes da sua conversão para digital. Estas mudanças foram realizadas com o intuito de diminuir o ruído apresentado no circuito original. A Figura 3 apresenta o diagrama de blocos do projeto atual. Por questões de confidencialidade, os circuitos elétricos do projeto não serão apresentados neste trabalho.

No desenvolvimento do projeto, inicialmente foi feita uma avaliação nos circuitos e sensores utilizados no projeto de Madureira \& Mendes (2007), para verificar quais deles poderiam ser aproveitados neste novo projeto. Concluiu-se que os sensores utilizados e o pré-amplificador presentes no projeto anterior poderiam ser utilizados neste novo projeto, com pequenas alterações no circuito pré-amplificador. Seguindo a linha do diagrama de blocos apresentado na Figura 3, após a análise inicial descrita, foram projetados e testados os filtros, amplificador e conversor A/D. Em seguida, pelas características necessárias ao projeto, foram escolhidos o PIC e o display utilizados. Por fim, foi realizada a programação do microcontrolador em questão. Tais circuitos e sensor encontram-se, brevemente, descritos a seguir.

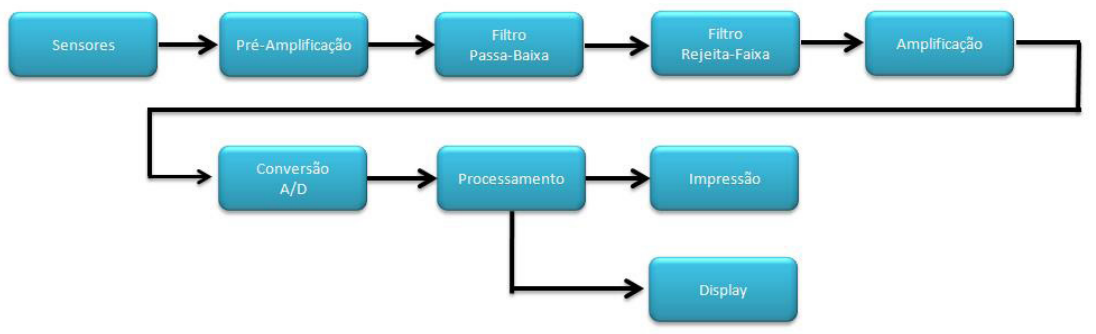

Figura 3 - Diagrama de Blocos do Projeto

\section{Sensor}

Sensores são dispositivos que transformam variáveis físicas de um processo (temperatura ou pressão, por exemplo), em variáveis convenientes à utilização em outro processo, como as variáveis elétricas (tensão, corrente, etc.) (Moraes \& Castricci, 2007). De acordo com Madureira \& Mendes (2007), os eletrodos de um eletrocardiógrafo são especiais para acoplar uma parte não metálica (corpo humano) a um circuito elétrico. Estes são muito utilizados em aplicações médicas, sendo responsáveis por realizar a interface entre um dispositivo eletrônico e a pele, podendo captar ou transmitir corrente elétrica.

Os eletrodos utilizados neste trabalho (do fabricante 3M) são do tipo adesivo. Este modelo foi escolhido por estar entre os mais utilizados, mundialmente, em eletrocardiografia. Além disso, ele dispensa o uso de gel para acoplamento e possui baixo custo, sendo fixado diretamente na pele do paciente. 


\section{Pré-amplificação}

O amplificador de instrumentação é o amplificador mais indicado para aplicações envolvendo sensores, pois este apresenta alta imunidade ao ruído de modo comum e, portanto, é a escolha natural para receber o sinal do sensor e fazer a primeira amplificação (ou pré-amplificação).

O amplificador de instrumentação escolhido para o projeto foi o mesmo utilizado por Madureira \& Mendes (2007): o INA114AP. O circuito utilizado foi implementado de acordo com o manual do seu fabricante. Este circuito difere do apresentado por Madureira \& Mendes (2007), pois foi acrescentado um circuito drive para a blindagem do cabo de interface, ligado diretamente ao eletrodo de referência, posicionado na perna direita: o right leg drive. Esse circuito atua realimentando o corpo com o sinal de modo comum com fase invertida, o que resulta na diminuição desse sinal, na entrada do amplificador de instrumentação (Macfarlane et al., 2011).

\section{Filtros}

A frequência dos sinais elétricos gerados pelo coração, de acordo com Macfarlane et al. (2011), não ultrapassa $250 \mathrm{~Hz}$. Logo, qualquer sinal com frequência superior a $250 \mathrm{~Hz}$ não faz parte do escopo do projeto e deve ser atenuado. Madureira \& Mendes (2007) utilizaram uma associação de dois filtros passa-baixa de segunda ordem para gerar um filtro de quarta ordem. Neste projeto foi utilizada a aproximação de Butterworth para gerar um filtro passa-baixa de quarta ordem.

Após o filtro passa-baixa, um filtro rejeita-faixa foi utilizado com o objetivo de minimizar os efeitos do ruído da rede elétrica $(60 \mathrm{~Hz})$.

\section{Amplificação}

Este estágio tem a função de adequar o sinal para a conversão A/D. Ao circuito amplificador, foi acoplado um circuito grampeador, responsável por adicionar um nível DC ao sinal amplificado, visando compatibilizá-lo com a entrada do conversor A/D (Malvino, 1995). A etapa de amplificação foi projetada para um ganho arbitrado em 10.

\section{Conversão Analógica/Digital}

Apesar de o $\mu \mathrm{C}$ adotado no projeto possuir 13 canais disponíveis para a conversão $\mathrm{A} / \mathrm{D}$, com resolução de 10 bits, o que garante 1024 níveis de conversão, esta resolução foi considerada insuficiente para garantir a performance gráfica ideal para o sinal. Assim, optou-se por utilizar um conversor de 12 bits do fabricante Microchip, modelo MCP3201.

O conversor A/D MCP3201 realiza até 100.000 amostras por segundo, quando operando com alimentação de $5 \mathrm{~V}$, mantendo um consumo máximo, quando ativo, de $400 \mu \mathrm{A}$. A grande vantagem deste CI é que ele se comunica com o $\mu \mathrm{C}$ através de protocolo serial SPI, ou seja, utilizando apenas três terminais. Devido a esta característica, o tamanho do circuito final é minimizado e economizam-se portas do $\mu \mathrm{C}$.

\section{Processamento do sinal}

$\mathrm{O} \mu \mathrm{C}$ escolhido para o cerne do projeto foi PIC18F4620. Os $\mu$ Cs PIC são baseados na arquitetura Harvard, com instruções do tipo RISC. Do total de 40 pinos, 35 podem ser configurados como entrada/saída (Miya- 
daira, 2009).

Este estágio do projeto recebe o sinal convertido em digital e gera um arquivo com os dados armazenados, referentes aos exames e ao cadastro dos clientes. Estes arquivos serão exibidos em um display TFT: thin-film transistor (transistor de película fina) e ainda poderão ser impressos, com auxílio de um software dedicado.

\section{Display}

Para a criação de uma interface microprocessada mais amigável, foi escolhido um módulo display LCD (display de cristal líquido) tipo TFT, com touch screen modelo SLCD43, do fabricante Reach Tech. Este módulo display possui uma placa controladora que embute o controle do painel LCD, um drive para o backlight e o touch screen. O display possui 4,3 polegadas, considerado adequado para a visualização e manipulação de um equipamento portátil. A resolução do display é de 480 por 272 pixels e o touch screen é resistivo.

A comunicação entre o display e o $\mu \mathrm{C}$ é realizada pela porta serial através do protocolo RS232. A placa controladora conta ainda com memória de 4 Mega bytes para gravação de imagens e macros.

\section{Filtros Digitais}

Mesmo com todas as precauções tomadas para diminuir a influência dos ruídos externos, a resposta na saída da placa analógica ainda apresentou ruídos, que poderiam prejudicar a interpretação do exame.

Para resolver esse problema, foram implementados filtros digitais do tipo Resposta de Impulso Infinita (IIR), diretamente no programa do $\mu \mathrm{C}$, logo após a conversão do sinal analógico para digital, realizada pelo MCP3201. Inicialmente, foram utilizados um filtro passa-baixa com frequência de corte de $200 \mathrm{~Hz}$ e três filtros notch com frequências centrais de 60,120 e $180 \mathrm{~Hz}$, que correspondem, respectivamente, à frequência da rede elétrica e seus segundo e terceiro harmônicos. Os resultados práticos mostraram que os melhores resultados foram obtidos utilizando-se apenas o filtro passa-baixa.

O filtro passa-baixa dessa etapa foi calculado utilizando-se um polinômio de Butterworth de segundo grau. A frequência de corte utilizada foi de $200 \mathrm{~Hz}$ e a frequência de amostragem foi de $500 \mathrm{~Hz}$. Os coeficientes do filtro analógico foram calculados com o auxílio do software Matlab. A função de transferência do filtro digital é mostra da na equação (1) (Tan \& Jiang, 2007), sendo a curva de resposta do filtro e a tela de comando do Matlab mostrados na Figura 4.

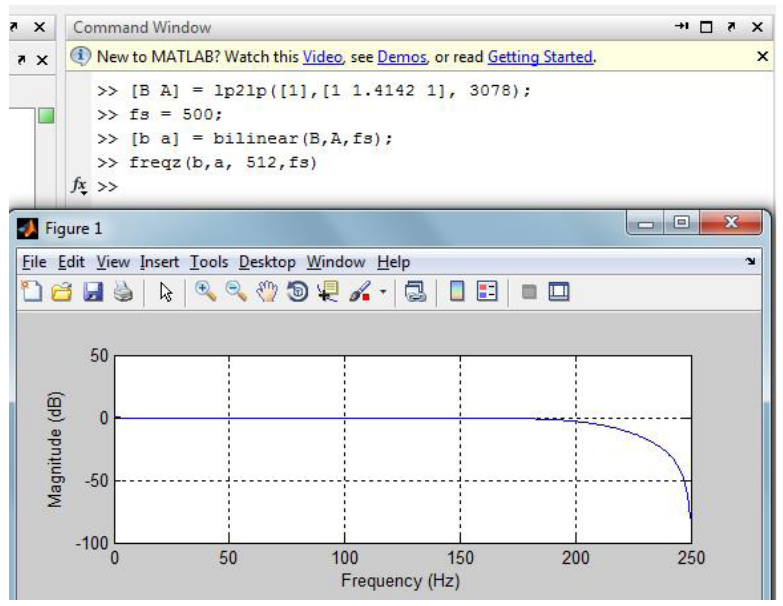

Figura 4 - Resposta em Frequência do Filtro Passa-Baixa e a Tela de Comando do Matlab. 


\section{Impressão}

Para que um médico efetue o laudo a partir de um eletrocardiograma, ele necessita receber um relatório adequado, que mostre, corretamente, a atividade elétrica do coração. Além da monitorização, o eletrocardiógrafo deve ser capaz de promover o registro, a impressão e o arquivamento dos exames realizados.

O eletrocardiógrafo desenvolvido armazena os dados do exame em arquivos de texto. Esses arquivos, na verdade, estão em formato XML, para facilitar a geração dos relatórios. Os dados do exame são gravados em um cartão de memória do tipo SD.

Para permitir a impressão e a visualização dos dados do exame, foi implementado um software escrito em Visual Basic, no compilador Visual Studio 2010 da Microsoft. O programa funciona gerenciando um banco de dados com informações, não apenas dos pacientes, mas também dos usuários envolvidos. Este banco de dados foi montado em formato Access, também da Microsoft. O Access foi escolhido pela sua interface visual, que o torna mais intuitivo e requer menor conhecimento específico.

\section{Resultados}

Os protótipos para cada estágio analógico foram montados, inicialmente, em placas separadas. Porém, em virtude da forte interferência na frequência de $60 \mathrm{~Hz}$, o protótipo foi alterado, implementando em uma única placa de circuito impresso, todos os circuitos analógicos do projeto (Figura 5). Foi adicionada uma blindagem metálica, cobrindo todo o circuito, para a prevenção contra interferências eletromagnéticas. Cada um dos cabos dos eletrodos possui uma malha de blindagem, também com o intuito de prevenir ruídos. Com isso, garantiu-se a diminuição dos ruídos em níveis aceitáveis.

O projeto dos filtros foi realizado com alto nível de criticidade e empregou amplificadores operacionais de baixo ruído. A Figura 6 mostra a resposta em frequência obtida na saída do filtro passa-baixa. A frequência de corte (ganho de $-3 \mathrm{~dB}$ ) está em torno de $220 \mathrm{~Hz}$.

Houve um problema de compatibilidade com o microcontrolador escolhido inicialmente. Nele, as portas de comunicação via USART e a porta de comunicação Serial SPI possuem um pino em comum. Tentou-se, sem sucesso, utilizar uma das duas portas via implementação por software, mas, a memória RAM do microcontrolador não era suficientemente grande para gerenciar o processo, e o componente entrava constantemente em reset. Não foi possível gerir a multiplexação deste pino. Para solucionar o problema, o PIC18F4550 foi substituído pelo PIC18F4620. Este último possui características similares ao adotado inicialmente, sem o problema verificado no componente anterior, não sendo necessário realizar mudanças significativas no código fonte desenvolvido.

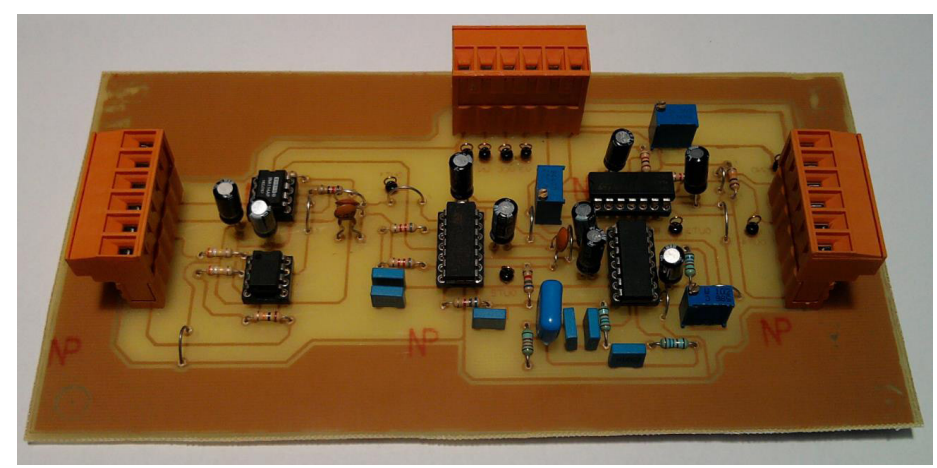

Figura 5 - Placa do circuito analógico 


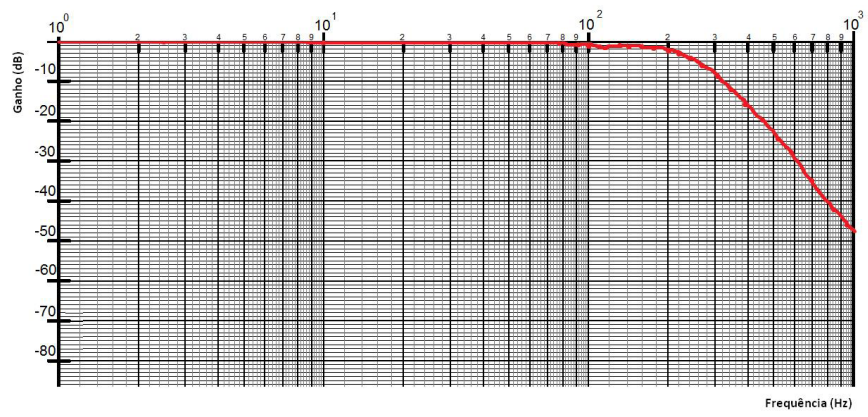

Figura 6 - Curva de Resposta Real do Filtro Passa-Baixa implementado no circuito.

Após as adaptações de cada etapa, o protótipo funcionou a contento. O custo total do projeto, incluindo mão de obra (prevista para a montagem de 200 peças) foi de aproximadamente $\mathrm{R} \$ 529,00$.

Um exemplo de forma de onda, obtido nos testes finais do eletrocardiógrafo projetado, é mostrado na Figura 7, em tela de LCD. A Figura 8 mostra um sinal coletado ao final dos testes do equipamento projetado por Madureira \& Mendes (2007), em tela de osciloscópio. Pode-se observar uma melhoria significativa na qualidade do sinal digital obtido nesse projeto (Figura 7), se comparado ao resultado de Madureira \& Mendes (Figura 8).

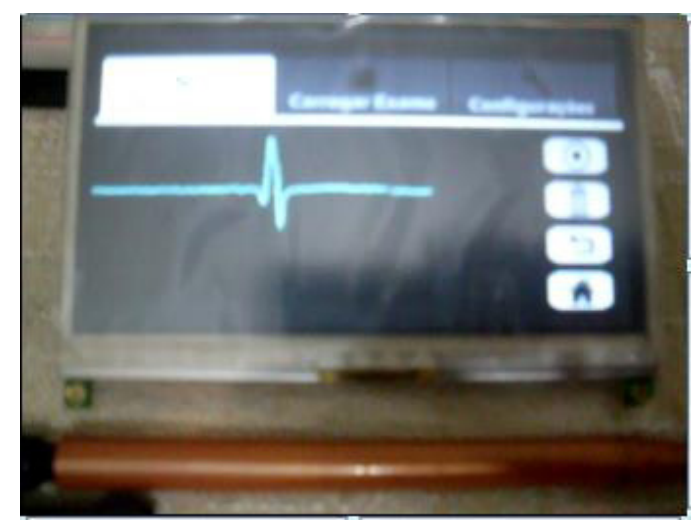

Figura 7 - Exemplo de um Eletrocardiograma em Tela de Display LCD.

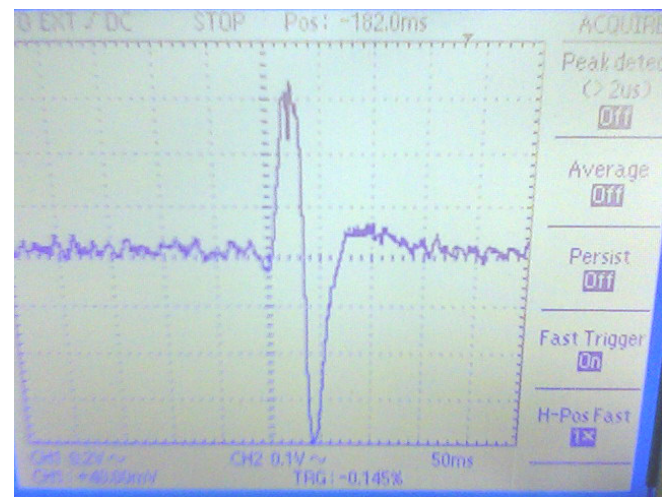

Figura 8 - Resultado Apresentado por Madureira \& Mendes (2007). 


\section{Discussões e Conclusões}

Este trabalho apresentou o projeto de um eletrocardiógrafo digital, desenvolvido a partir do eletrocardiógrafo analógico projetado por Madureira \& Mendes (2007). Trata-se de um eletrocardiógrafo portátil, construído com baixo custo e que possui apresentação em display LCD, com tecnologia touch screen, e que é capaz de gravar e ler exames em cartão SD.

Em teste práticos, constatou-se que o eletrocardiógrafo produziu resultados satisfatórios, sendo o seu funcionamento adequado, de acordo com a expectativa da área médica. Todos os circuitos implementados, após ajustes, funcionaram a contento. A utilização do filtro digital recursivo foi fundamental para assegurar que os ruídos da fonte de alimentação e do ambiente fossem minimizados, dando condições para que o sinal seja interpretado de maneira correta.

As Figuras 7 e 8 evidenciam uma melhoria significativa na qualidade do sinal digital obtido neste projeto, em relação às formas de onda obtidas por Madureira \& Mendes (2007).

Em relação aos equipamentos disponíveis no mercado, como o ECG PC e o Cardiocare 2000, por exemplo, o eletrocardiógrafo desenvolvido, além do custo mais baixo (os citados estão na faixa de $\mathrm{R} \$ 3.000,00$ ), possui a vantagem de possuir interface em touch screen, o que possibilita maior facilidade de interação com $o$ usuário.

O desenvolvimento de uma plataforma intuitiva possibilita a gravação do exame sem exigir conhecimentos avançados sobre o equipamento. Outra vantagem do sistema é que ele faz a gravação dos arquivos em cartão de memória do tipo SD, que atualmente é um formato largamente difundido.

O instrumento projetado pode ser construído com custo financeiro relativamente baixo (em torno de R\$ 500,00 ), atingindo a meta do trabalho, mesmo com a utilização de amplificadores operacionais de baixo ruído e com um módulo display microprocessado.

Pode-se concluir, portanto, que o projeto desenvolvido alcançou os objetivos propostos, pois permitiu adquirir o sinal elétrico do coração, apresentando-o em formato digital, em um display de cristal líquido com tela sensível ao toque. O eletrocardiógrafo desenvolvido possui baixo custo e, além de possibilitar a visualização do sinal digital, permite que o exame seja gravado em um cartão do tipo SD, para posterior análise. O software computacional, também implementado neste projeto, permite que o exame realizado seja impresso. O equipamento desenvolvido também pode ser alimentado por baterias, o que possibilita a portabilidade do produto.

Para que o projeto apresentado se torne um produto final, alguns aperfeiçoamentos devem ser realizados, tais como, a miniaturização do circuito, utilizando componentes de tecnologia de montagem superficial (Surface-Mount Technology - SMD), a utilização de um isolador galvânico, para aumentar a segurança do paciente e algumas melhorias no software para o computador, como aperfeiçoar o design do programa, facilitar, ainda mais, o acesso ao arquivo do exame e permitir a geração do relatório do exame, sem a necessidade de conexão com a internet.

\section{Referências Bibliográficas}

ACHARYA, U. R.; SURI, J. S.; SPAAN, J. A. E.; KRISHNAN, S. M. Advances in Cardiac Signal Processing. Berlin: Springer, 2007.

CLAYTON, G.; WINDER, S. Operational Amplifiers. 5 ed. Oxford: Newnes, 2003.

HUANG, H. W. Pic Microcontroller: An Introduction to Software and Hardware Interfacing. Mankato: Thonson, 2005.

MACFARLANE, P. W.; OOSTEROM, A. V.; PAHLM, O.; KLIGFIELD, P.; JANSE, M.; CAMM, J. Comprehensive Electrocardiology. 2 ed. London: Springer, 2011.

MADUREIRA, A. L. A.; MENDES, F. S. Eletrocardiógrafo de Baixo Custo. 2007. Monografia (Engenharia Elétrica) - Universidade Gama Filho, Rio de Janeiro.

MALVINO, A. P. Eletrônica: Volume 1. 4 ed. São Paulo: Makron Books, 1995.

MIYADAIRA, A. N. Microcontroladores PIC18: Aprenda e Programe em Linguagem C. 1 ed. São Paulo: Érica, 2009.

MORAES, C. C.; CASTRUCCI, P. L. Engenharia de Automação Industrial. 2 ed. Rio de Janeiro: LTC, 2007. 